\title{
Predictive model of increased mortality and bed occupancy if thrombolysis becomes the initial treatment strategy for STEMI during the SARS-CoV-2 pandemic
}

\author{
Authors: Luke Dancy ${ }^{A}$, Kevin O'Gallagher ${ }^{B}$, Matthew Ryan ${ }^{C}$, Philip A MacCarthy ${ }^{D}$ and Ajay M Shah ${ }^{E}$
}

\begin{abstract}
During the current SARS-CoV-2 pandemic the restructure of healthcare services to meet the huge increase in demand for hospital resource and capacity has led to the proposal that where necessary ST elevation myocardial infarction (STEMI) could be managed by intravenous thrombolysis in the first instance as a means of reducing the workforce requirements of a primary angioplasty service run at a heart attack centre. Our modelling, based on data from the UK, shows that contrary to reducing demand, the effect on both mortality and bed occupancy would be negative with 158 additional deaths per year for each $10 \%$ reduction in primary angioplasty and at a cost of $\sim 8,000$ additional bed days per year for the same reduction. Our analysis demonstrates that specialist services such as heart attack pathways should be protected during the COVID crisis to maximise the appropriate use of resource and prevent unnecessary mortality.
\end{abstract}

KEYWORDS: STEMI, COVID-19, primary PCI, thrombolysis

DOI: $10.7861 /$ clinmed.2020-0293

\section{Background}

The SARS-CoV-2 pandemic represents an extreme challenge to healthcare systems globally. Of patients admitted to hospital with COVID-19, up to a quarter require management in intensive care units (ICUs). ${ }^{1-4}$ ICU capacity to cope with this surge in demand is a major problem, not only in the UK, which has a relatively low number of ICU beds ( 6.6 beds $/ 100,000$ population), ${ }^{5}$ but also in

Authors: Aregistrar in interventional cardiology, King's College Hospital NHS Foundation Trust, London, UK; B Medical Research Council clinical research fellow, The King's College London British Heart Foundation Centre of Research Excellence, London, UK; 'British Heart Foundation clinical research fellow, The King's College London British Heart Foundation Centre of Research Excellence, London, UK; Dprofessor of cardiology, King's College Hospital NHS Foundation Trust, London, UK; Eprofessor of cardiology, The King's College London British Heart Foundation Centre of Research Excellence, London, UK high-capacity countries such as the USA $(33.6 / 100,000) .{ }^{6}$ Reducing demand on ICUs from non-COVID-19 related conditions, such as elective operations, is therefore essential. Furthermore, overall hospital bed capacity needs modification to accommodate patients with less severe COVID-19 disease and to create temporary ICUs. Consequently, hospitals have considered ways to change the way they manage conditions such as ST-elevation myocardial infarction (STEMI).

The gold-standard treatment for STEMI is urgent transfer to heart attack centres (HACs) that provide 24/7 primary percutaneous coronary intervention (PPCI). Current discussions, and indeed recommendations in some countries, have proposed that intravenous thrombolysis could be employed instead of PPCI as the initial management for STEMI in the event of insufficient staff numbers, either due to redeployment or illness. The heterogenous nature of non-ST elevation myocardial infarction presentations and the lack of need for emergency intervention make considerations surrounding NSTEMI more complex and beyond the scope of this paper. Thrombolysis reduces the initial demand for expert staff and patient transfer to HACs and potentially exposes fewer healthcare workers to a COVID-infected patient (the usual team for PPCI comprises operator, scrub nurse, running nurse, radiographer and physiologist). Personal protective equipment (PPE) requirements based on current recommendations would be two operators and scrub nurse wearing full PPE and the remainder of the team in moisture resistant surgical masks and gown and gloves.

\section{Predicting the impact of changing from PPCI to} thrombolysis as initial management for STEMI

Although thrombolysis is superior to placebo, meta-analysis of randomised controlled trials has shown PPCI is significantly better, achieving reductions in major adverse cardiac and cerebrovascular events (MACCE) and with long-term benefit. ${ }^{7}$ Mortality was $7 \%$ after PPCI versus $9 \%$ after thrombolysis $(p=0.0002)$ and $3 \%$ suffered recurrent ischaemia compared to $7 \%(P<0.0001)$. The increased mortality also serves as a surrogate for ICU occupation, given that many inpatient STEMI deaths involve ICU admission. In real-world data from the Myocardial Ischaemia National Audit Project (MINAP), ${ }^{8}$ which captures all acute treatment procedures for MI in England, the mortality from STEMI between 2015-2018 
a Management strategies of patients presenting with STEMI in the UK in 2017/18 (\%)

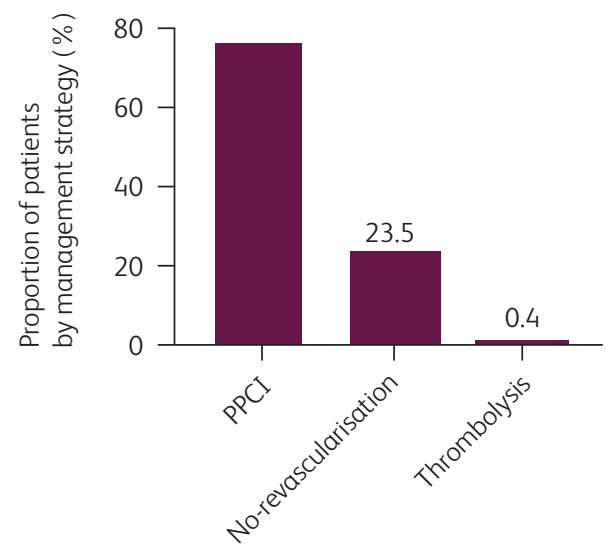

b Estimated mortality change in STEMI with decreasing PPCI application

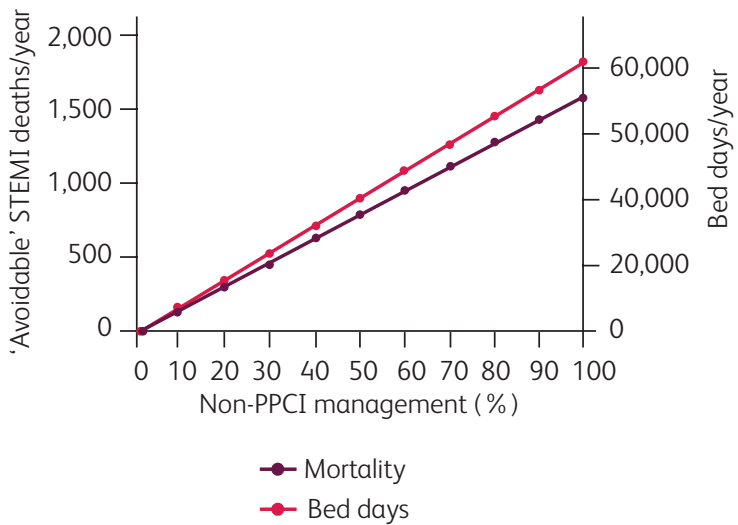

Fig 1. Estimated impact of altering management strategy for primary percutaneous coronary intervention (PPCI) in the UK. a) Initial management strategy for STEMI in the UK in 2018 ( $n=35,740)$. b) Predicted increase in mortality and bed day numbers with progressive increases in thrombolysis instead of PPCI for treatment of STEMI in the UK. Based on 30-day STEMI mortality in England over 3 years to 2018. Bed day calculation based on 4-day admission for PPCI-managed STEMI and 7 day for non-PPCI managed STEMI. Data from Myocardial Ischaemia National Audit Project. ${ }^{8}$

was $9.35 \%(n=77,604)$ where PPCI was available and $15.14 \%$ $(n=9,084)$ when patients were admitted initially to a non-PPCI centre, regardless of whether they were eventually transferred for revascularisation. The vast majority of patients were revascularised by PPCI (Fig 1a).

Based on these data, we estimated the impact of changing from PPCI to thrombolysis as initial management for STEMI in the UK, assuming that the characteristics of the overall patient population remain unchanged. Applying these figures to the numbers of PPCI cases in the UK in 2018 , each $10 \%$ reduction in PPCI equates to approximately 158 'avoidable' deaths per year (Fig 1b). We next estimated the impact of changing management strategies on the inpatient length of stay. Currently, low-risk, uncomplicated STEMI patients managed using PPCI are discharged 72 hours post-event. ${ }^{9}$ The admission duration for thrombolysed STEMI is longer. Furthermore, since only $25-40 \%$ of patients achieve TIMI-3 flow with lysis alone and $10 \%$ will reinfarct during their stay, ${ }^{10}$ it is unlikely there would be a significant reduction in the requirement for invasive angiography. Also, given the increased complication rates in the thrombolysis group, a greater proportion may need ICU care while revascularisation is planned. Considering only uncomplicated STEMI presentations, each $10 \%$ increase in the number of patients managed with thrombolysis followed by angiography/PCI would lead to 8000 additional bed days in the UK in a single year (using a conservative median admission time of 4 days for PPCI and 7 for thrombolysed STEMIs) - see Fig 1b.

\section{Effect of COVID-19 on presentations}

There are additional considerations specific to the current pandemic. PPCI is superior to thrombolysis in late-presenting myocardial infarction. The proportion of late presentations increased during the first wave of the COVID-19 pandemic. Data from Italy demonstrated a $25 \%$ increase in late presentations during the peak of their outbreak.11 Data from a UK centre report a 33\% decrease in STEMI presentations, but a significant increase in late-presentation of STEMI (time from symptoms to first medical contact $>12$ hours in $26 \%$ of patients). Furthermore, serum troponin levels on presentation were higher than in an equivalent pre-COVID-19 time period, suggesting increased magnitude of myocardial necrosis on presentation. ${ }^{12}$ The converse scenario is also possible: observational data from China and Italy indicate that some patients with COVID-19 present with apparent ST elevation acute coronary syndromes but without coronary occlusion; some may have myocarditis. Thrombolysis in such patients may cause significant harm whereas invasive angiography can expediently rule out occlusive coronary disease. Thrombolysis could therefore be used where it is least effective or not needed respectively.

\section{Conclusion}

It is clear that this global health crisis has mandated a seismic, but hopefully temporary, shift in the management of patients requiring inpatient care to preserve resources needed for those with severe COVID-19. Healthcare professionals had to be redeployed to areas outside their usual roles. Great care needs to be taken, however, to ensure that patients presenting with other serious, often life-threatening, conditions such as STEMI continue to receive appropriate, guideline-driven therapy. Different solutions may be required in different settings, eg highly efficient protected pathways for PPCI within larger specifically designated HACs where interventional cardiology capacity and staffing are maintained.

Whatever the local solution, our analysis indicates that changing to an initial strategy of thrombolysis instead of PPCI could significantly increase mortality of patients who would otherwise have a good prognosis, as well as placing additional strain on the very departments and facilities that we are trying to maintain to tackle the COVID-19 crisis either currently or in the event of future waves of infection. 


\section{References}

1 Chen N, Zhou M, Dong X et al. Epidemiological and clinical characteristics of 99 cases of 2019 novel coronavirus pneumonia in Wuhan, China: a descriptive study. Lancet 2020;395:507-3.

2 Bean DM, Kraljevic Z, Searle T et al. ACE-inhibitors and angiotensin-2 receptor blockers are not associated with severe SARSCOVID19 infection in a multi-site UK acute Hospital Trust. Eur ] Heart Fail 2020, in press (doi: 10.1002/ejhf.1924).

3 Grasselli G, Pesenti A, Cecconi M. Critical care utilization for the COVID-19 outbreak in Lombardy, Italy: early experience and forecast during an emergency response. JAMA 2020, in press (doi: 10.1001/jama.2020.4031).

4 Cummings MJ, Baldwin MR, Abrams D et al. Epidemiology, clinical course, and outcomes of critically ill adults with COVID-19 in New York City: a prospective cohort study. Lancet 2020;395:1763-70.

5 Rhodes A, Ferdinande P, Flaatten $\mathrm{H}$ et al. The variability of critical care bed numbers in Europe. Intensive Care Med 2012;38:1647-53.

6 Society of Critical Care Medicine. Critical care statistics. www.sccm. org/Communications/Critical-Care-Statistics.

7 Keeley EC, Boura JA, Grines CL. Primary angioplasty versus intravenous thrombolytic therapy for acute myocardial infarction: a quantitative review of 23 randomised trials. Lancet 2003;361:13-20.

8 National Institute for Cardiovascular Outcomes Research. Myocardial Ischaemia National Audit Project: 2019 summary report (2017/18 data). NICOR, 2019. www.nicor.org.uk/wp-content/ uploads/2019/09/MINAP-2019-Summary-Report-final.pdf.

9 Swaminathan RV, Rao SV, McCoy LA et al. Hospital length of stay and clinical outcomes in older STEMI patients after primary PCI: a report from the National Cardiovascular Data Registry. J Am Coll Cardiol 2015;65:1161-71.

10 de Belder MA. Coronary disease: acute myocardial infarction: failed thrombolysis. Heart 2001;85:104-12.

11 Roffi M, Guagliumi G, Ibanez B. The obstacle course of reperfusion for ST-segment-elevation myocardial infarction in the COVID-19 pandemic. Circulation 2020;141:1951-3.

12 Abdelaziz HK, Abdelrahman A, Nabi A et al. Impact of COVID-19 pandemic on patients with ST-segment elevation myocardial infarction: Insights from a British cardiac center. Am Heart J 2020;226:45-8.

Address for correspondence: Dr Kevin O'Gallagher, The King's College London British Heart Foundation Centre of Research Excellence, School of Cardiovascular Medicine \& Sciences, The James Black Centre, 125 Coldharbour Lane, London SE5 9NU, UK.

Email: kevin.o'gallagher@kcl.ac.uk 